\title{
Enhanced screening and spectral diversity in many-body elastic scattering of excitons in two-dimensional hybrid metal-halide perovskites
}

\author{
Félix Thouin, ${ }^{1}$ Daniele Cortecchia, ${ }^{2}$ Annamaria Petrozza, ${ }^{2}$ Ajay Ram Srimath Kandada, ${ }^{2}$ and Carlos Silva $\odot^{1,3,4, *}$ \\ ${ }^{1}$ School of Physics, Georgia Institute of Technology, 837 State Street NW, Atlanta, Georgia 30332, USA \\ ${ }^{2}$ Center for Nano Science and Technology@ PoliMi, Istituto Italiano di Tecnologia, via Giovanni Pascoli 70/3, 20133 Milano, Italy \\ ${ }^{3}$ School of Chemistry and Biochemistry, Georgia Institute of Technology, 901 Atlantic Drive NW, Atlanta, Georgia 30332, USA \\ ${ }^{4}$ School of Materials Science and Engineering, Georgia Institute of Technology, North Avenue, Atlanta, Georgia 30332, USA
}

(Received 11 June 2019; published 4 December 2019)

\begin{abstract}
We quantify the role of many-body elastic scattering effects on exciton dephasing rates in two-dimensional hybrid metal-halide perovskites by means of nonlinear coherent excitation spectroscopy at a temperature of $5 \mathrm{~K}$. We find that the exciton-density dependence of excitation-induced dephasing (EID) is two to three orders of magnitude lower than in other atomic monolayer semiconductors such as transition metal dichalchogenides. Furthermore, EID is different for the multiple excitons evident in the excitation line shape, as is their phononmediated temperature dependence. We ascribe these observations to screening effects due to polaronic dressing by the lattice.
\end{abstract}

DOI: 10.1103/PhysRevResearch.1.032032

In two-dimensional hybrid organic-inorganic metal-halide perovskites, the intrinsic optical line shape reflects multiple excitons with distinct binding energies [1,2], each dressed differently by the hybrid lattice [3]. Given this complexity, a fundamentally far-reaching issue is how Coulomb-mediated many-body interactions-elastic scattering such as excitationinduced dephasing [4], inelastic exciton bimolecular scattering [5], and multiexciton binding [6,7]-depend upon the specific exciton-lattice coupling. We report the intrinsic and density-dependent exciton pure dephasing rates and their dependence on temperature by means of a coherent nonlinear spectroscopy. We find exceptionally strong screening effects on multiexciton scattering relative to other two-dimensional single-atomic-layer semiconductors. Importantly, the excitondensity dependence of the dephasing rates is markedly different for distinct excitons. These findings establish the consequences of particular lattice dressing on exciton many-body quantum dynamics, which critically define fundamental optical properties that underpin photonics and quantum optoelectronics in relevant exciton density regimes.

Spectral transition linewidths provide pertinent insights into the system-bath interactions in materials because they depend on optical dephasing dynamics - the processes by which the coherence that the driving electromagnetic wave imparts on the optical response dissipates due to scattering processes with lattice phonons, other excitations, and defects.

\footnotetext{
*carlos.silva@gatech.edu

Published by the American Physical Society under the terms of the Creative Commons Attribution 4.0 International license. Further distribution of this work must maintain attribution to the author(s) and the published article's title, journal citation, and DOI.
}

Dephasing rates thus are very sensitive probes of the consequences of lattice dressing effects on excitons. Nevertheless, these are challenging to extract directly from linear optical probes such as absorption or photoluminescence spectroscopy given that the experimental linewidths typically arise from two distinct but coexisting contributions: homogenous and inhomogenous broadening [see Fig. 1(a)]. While the former is due to dephasing and is governed by the intrinsic finite lifetime of excited states and by dynamic disorder, the latter is caused by a statistical distribution of the transition energy due to static disorder, defects, or grain boundaries. The exciton homogeneous linewidth $2 \gamma$ (full width at half maximum) is limited by the exciton lifetime $\left(\Gamma^{-1}\right)$ and the dephasing rate mediated by exciton-exciton elastic scattering (excitation-induced dephasing, $\gamma_{\text {EID }} / \hbar$ ) and phonon scattering $\left(\gamma_{\mathrm{ph}} / \hbar\right)$ :

$$
\gamma=\frac{\hbar \Gamma}{2}+\gamma_{\mathrm{EID}}+\gamma_{\mathrm{ph}}
$$

An accurate estimate of $\gamma$ is thus crucial to quantify the magnitude of the interexciton and exciton-phonon scattering cross sections, which, in turn, influences the radiative rates and transport characteristics. Exciton-density-dependent transient photobleach linewidth analysis has been applied to address time-dependent lineshapes in a two-dimensional (2D) hybrid perovskite [8]. However, it is challenging to separate homogeneous dephasing effects from other $\vec{k}$-space-filling density effects by this means, and therefore to rigorously quantify $\gamma$. Here, we implement nonlinear coherent spectroscopy to unambiguously extract the homogeneous dephasing rates of the various excitonic transitions in a polycrystalline thin film of single-layered $(\mathrm{PEA})_{2} \mathrm{PbI}_{4}(\mathrm{PEA}=$ phenylethylammonium). Figure 1(b) shows the linear absorption spectrum measured at $5 \mathrm{~K}$. Four distinct excitons (labeled A, $\mathrm{A}^{\prime}, \mathrm{B}$, and $\mathrm{B}^{*}$ ) are observed about $200 \mathrm{meV}$ below the continuum band edge 

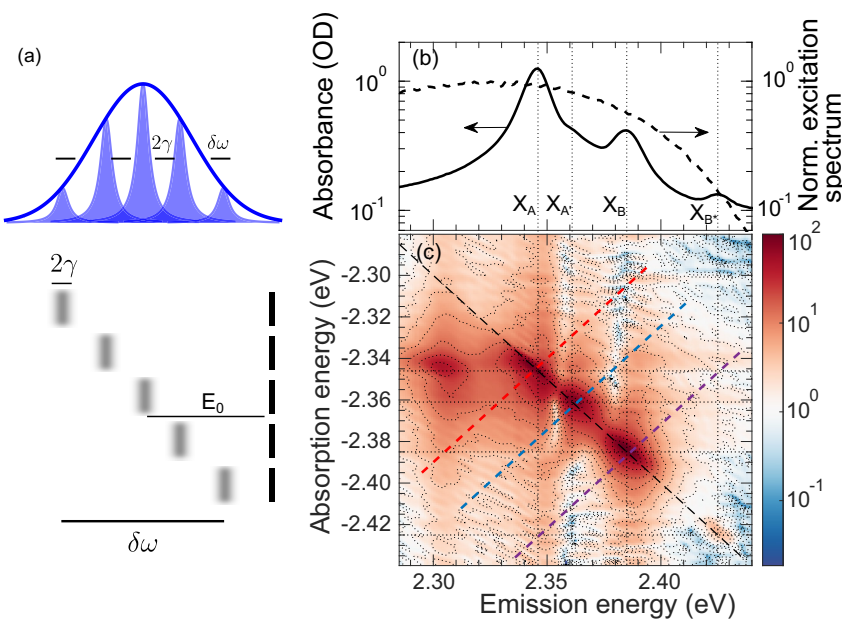

FIG. 1. Linear and two-dimensional coherent spectroscopy of (PEA) ${ }_{2} \mathrm{PbI}_{4}$ at $5 \mathrm{~K}$. (a) Illustration of the inhomogeneous and homogeneous nature of line broadening. The total linewidth (blue line) is composed of a distribution of homogeneously broadened lines (blue areas). This arises from the simultaneous existence of uncorrelated transitions from the ground state (black lines) to short-lived excited states (blurred lines). $E_{0}$ represents the central energy, and $\delta \omega$ and $2 \gamma$ are the inhomogeneous and homogeneous linewidths respectively. (b) Absorption spectrum of $(\mathrm{PEA})_{2} \mathrm{PbI}_{4}$ measured at $5 \mathrm{~K}$ (black line) and normalized spectrum of the pulses used in $2 \mathrm{D}$ coherent excitation spectroscopy measurements (dashed line). Both scales are logarithmic. Dotted lines indicate the energy of excitons A, A', B, and $\mathrm{B}^{*}$, respectively, with increasing energy. (c) Absolute value of the $2 \mathrm{D}$ coherent rephasing spectrum of $(\mathrm{PEA})_{2} \mathrm{PbI}_{4}$ measured at $5 \mathrm{~K}$ with a pulse fluence of $40 \mathrm{~nJ} / \mathrm{cm}^{2}$ and a pump-probe delay of $20 \mathrm{fs}$. The color scale is logarithmic. Dotted lines indicate the energies of the aforementioned features. The paths of the diagonal cut (black dashed line) and antidiagonal cuts at the diagonal energy of excitons A, A', and B (red, blue, and purple dashed lines, respectively) are also shown.

(the temperature-dependent absorption spectra are presented as Supplemental Material [9]). We quantify $\gamma$ as a function of exciton density and temperature and find that these different excitons display peculiar many-body phenomena.

Four-wave-mixing spectroscopies measure coherent emission due to a third-order polarization induced in matter by a sequence of phase-locked femtosecond pulses, and its dissipation reports directly on dephasing processes. In a two-dimensional coherent excitation geometry, one spectrally resolves this signal, and a 2D spectral correlation map of excitation and emission energies is constructed by time resolving the four-wave-mixing spectrum along a coherent excitation time variable and by Fourier transformation of the resulting coherence decay function. The absorption-emission diagonal axis thus contains the individual exciton resonances identified in the linear absorption spectrum, with any off-diagonal cross peaks displaying spectral correlations between them. With socalled rephasing phase matching, this 2D line shape permits separation of the homogeneous and inhomogeneous contributions to the optical linewidth. Specifically, if the homogeneous and inhomogeneous linewidths are comparable, which is the case for $(\mathrm{PEA})_{2} \mathrm{PbI}_{4}$ [2,7], the antidiagonal line shape is
$[10,11]$

$$
S_{A D}\left(\omega_{a d}\right)=\left|\frac{\exp \left(\frac{\left(\gamma-i \omega_{a d}\right)^{2}}{2 \delta \omega^{2}}\right) \operatorname{erfc}\left(\frac{\left(\gamma-i \omega_{a d}\right)}{\sqrt{2} \delta \omega}\right)}{\delta \omega\left(\gamma-i \omega_{a d}\right)}\right|,
$$

while the diagonal one reads as

$$
\begin{aligned}
S_{D}\left(\omega_{d}\right)= & \sum_{j} \alpha_{j} \mid \frac{\exp \left(\frac{\left[\gamma-i\left(\omega_{d}-\omega_{j}\right)\right]^{2}}{2 \delta \omega^{2}}\right)}{\gamma \delta \omega}\left[\operatorname{erfc}\left(\frac{\left[\gamma-i\left(\omega_{d}-\omega_{j}\right)\right]}{\sqrt{2} \delta \omega}\right)\right. \\
& \left.+\exp \left(\frac{2 \gamma i\left(\omega_{d}-\omega_{j}\right)}{\delta \omega^{2}}\right) \operatorname{erfc}\left(\frac{\left[\gamma+i\left(\omega_{d}-\omega_{j}\right)\right]}{\sqrt{2} \delta \omega}\right)\right] \mid
\end{aligned}
$$

Here erfc corresponds to the complementary error function, $\omega_{a d}$ and $\omega_{d}$ are the antidiagonal and diagonal angular frequencies, respectively, and $\delta \omega$ characterizes the inhomogeneous distribution. The sum in Eq. (3) runs over the relative amplitudes $\alpha_{j}$ and the central diagonal energies $\omega_{j}$ of optical transitions of excitons $\mathrm{A}, \mathrm{A}^{\prime}, \mathrm{B}$, and $\mathrm{B}^{*}$.

The laser spectrum used in this experiment, which covers all excitonic absorption features, is displayed in Fig. 1(b). The early-population-time 2D map of the absolute value of the rephasing signal from $(\mathrm{PEA})_{2} \mathrm{PbI}_{4}$ at $5 \mathrm{~K}$, population waiting time of $20 \mathrm{fs}$ [12], and a fluence of $40 \mathrm{~nJ} / \mathrm{cm}^{2}$ (excitation density $8.8 \times 10^{18} \mathrm{~cm}^{-3}$ or $3.5 \times 10^{13} \mathrm{~cm}^{-2}$ ) is shown in Fig. 1(c). Details about the experimental conditions are presented as Supplemental Material [9]. Four features are observed elongated along the diagonal axis, corresponding to excitons $\mathrm{A}, \mathrm{A}^{\prime}, \mathrm{B}$, and $\mathrm{B}^{*}$. Faint cross peaks between all excitonic features are also observed, indicating that all the transitions share a common ground state. An intense cross peak at an emission energy of $2.305 \mathrm{eV}$ is observed for exciton A despite the presence of a much weaker diagonal feature at lower energy than that dominant peak. Given the opposite phase of this feature (the real part of the spectra shown in Supplemental Materials [9]) when compared to the corresponding diagonal peak, we attribute it to an excitedstate absorption from a singly bound A exciton to a bound AA biexciton [7]. The evolution of this line shape with population waiting time (not shown in this article) is complex and likely displays the role of dark states, which will be treated in a separate publication.

By simultaneously fitting the diagonal cut and the antidiagonal cuts across the maximum of each diagonal features to the model described by Eqs. (2) and (3), we can extract the homogeneous and inhomogeneous linewidths of each excitons. The diagonal and antidiagonal cuts at the energies of excitons A, A', and B, along with the best fits to Eqs. (3) and (2), are plotted in Figs. 2(a)-2(c) respectively. The only fit parameters are the amplitudes $\alpha_{j}$, homogeneous dephasing width $\gamma$, and inhomogeneous width $\delta \omega$ of each optical transition. More information regarding the fitting procedure is presented in the Supplemental Material [9].

To assess the contribution of many-body interactions on the dephasing dynamics of the different excitons, we acquired 2D coherent excitation spectra for a wide range of excitation fluences and sample temperatures (the raw data are presented as Supplemental Material [9]). The monotonic rise of $\gamma$ with the 

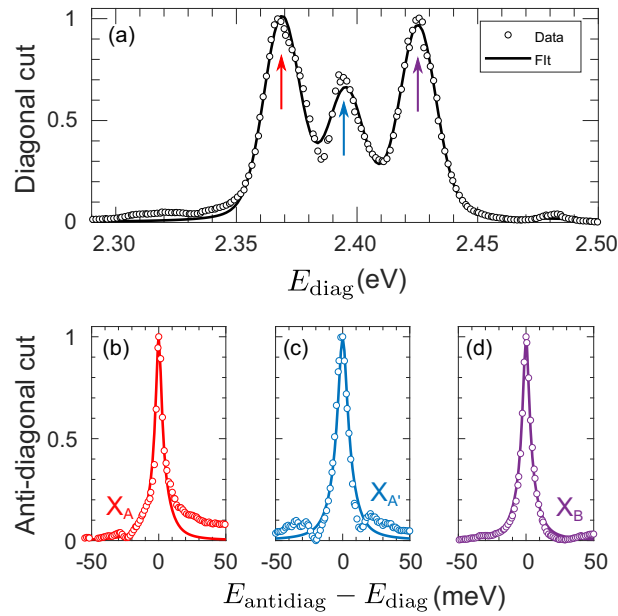

FIG. 2. Fitting diagonal and antidiagonal spectral cuts to a lineshape model. Normalized diagonal cut (a) and antidiagonal cuts at diagonal energies of excitons $\mathrm{A}, \mathrm{A}^{\prime}$, and $\mathrm{B}$ [panels (b)-(d), respectively) of the two-dimensional coherent excitation rephasing spectrum presented in Fig. 1(c) (circles) and the result of the fitting procedure described in the main text (lines). Arrows in panel (a) mark the position along the diagonal where the antidiagonal cuts cross it. Because of the presence of low-amplitude cross peaks in the $2 \mathrm{D}$ spectrum, these also appear in the tails of the antidiagonal cuts. To minimize their effect on the quality of the fits and to avoid overestimation of the linewidths, only points higher than $15 \%$ of an antidiagonal cut maxima were included in the fit. The quality of the fits presented here are representative of all the presented dataset. To test the robustness of the fits and to estimate the uncertainties on the extracted linewidths, we repeated the fitting procedure numerous times while adding white noise ( $5 \%$ of the cut's maximum peak to peak) to the data.

excitation fluence at $5 \mathrm{~K}$ is shown in Fig. 3. Such a dependence on exciton density $n$ is a consequence of broadening induced by exciton-exciton elastic scattering mediated by long-range Coulomb interactions:

$$
\gamma_{\mathrm{EID}}(n)=\gamma_{0}+\Delta n
$$

Here, $\gamma_{0}$ is the density-independent dephasing rate and $\Delta$ is the exciton-exciton interaction parameter [4,10,11]. Excitons in 2D metal-halide perovskites are confined to one of the inorganic quantum wells and are electronically isolated from the others due to the large inter-layer distance [3] $(\approx 8 \AA)$ imposed between them by the long organic cations. However, the sample itself, $40 \mathrm{~nm}$ thick, is composed of tens of these quantum wells, leading to a highly anisotropic excitonexciton interaction. To allow for some form of comparison with other 2D semiconductors and quasi-two-dimensional quantum wells of similar thicknesses, we report the excitonexciton interaction parameter, $\Delta$ in units of energy per area. The associated fits and the fit parameters are displayed in Figs. 3(a)-3(c). While $\gamma_{0}$ is approximately $2 \mathrm{meV}$ with modest variation across the three probed excitonic transitions, $\Delta$ varies more substantially. It is $2.7 \times 10^{-12} \mu \mathrm{eV} \mathrm{cm}{ }^{2}$ for exciton $\mathrm{B}$, increases to $4.3 \times 10^{-12} \mu \mathrm{eV} \mathrm{cm}{ }^{2}$ for exciton $\mathrm{A}$, and to $8.6 \times 10^{-12} \mu \mathrm{eV} \mathrm{cm} \mathrm{cm}^{2}$ for exciton $\mathrm{A}^{\prime}$. Furthermore, for all excitons, $\gamma$ is consistently smaller with linearly polarized excitation than with circularly polarized pulses [9]. Moreover,

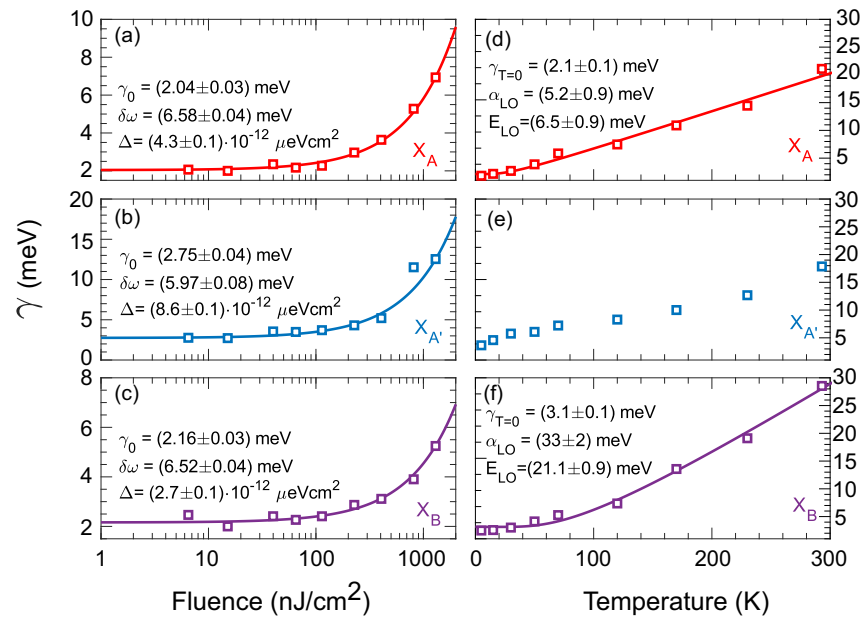

FIG. 3. Fluence and temperature dependence of the exciton dephasing rates. Dephasing parameters $\gamma$ of excitons A, $\mathrm{A}^{\prime}$, and B [panels (a) and (d), (b) and (e), and (c) and (f), respectively) obtained from the simultaneous fitting of diagonal and antidiagonal cuts, plotted as a function of excitation fluence [panels (a)-(c)] or temperature [panels (d)-(f)]. Squares represent the experimental linewidths and lines are the best fit to the relevant model described in the main text. Error bars on the data are contained within the markers. For panels (a)-(c), the sample's temperature is maintained at $5 \mathrm{~K}$ while the excitation fluence is kept at $50 \mathrm{~nJ} / \mathrm{cm}^{2}$ for measurements presented in panels (d)-(f).

except for exciton $\mathrm{A}^{\prime}$, it is independent of the helicity of the exciting pulses within our experimental uncertainty [9]. When exciting the sample with linearly polarized pulses, excitons can scatter on both left and right circularly polarized excitons. However, they can only scatter with excitons of the same polarization when excited with circularly polarized pulses due to conservation of angular momentum [13].

It is not straightforward to compare these values with those of other materials due to the ambiguities over the relevant values of the permittivity function and thus the Bohr radii. However, given the two-dimensional nature of the exciton and comparable exciton binding energies, monolayers of transition metal-dichalchogenides (TMDCs) provide a realistic benchmark. Intriguingly, previous 2D coherent excitation measurements on unencapsulated $\mathrm{WSe}_{2}$ [4] and encapsulated $\mathrm{MoSe}_{2}$ [14] revealed $\Delta=2.7 \times 10^{-12} \mathrm{meV} \mathrm{cm}^{2}$ and $4 \times$ $10^{-13} \mathrm{meV} \mathrm{cm}^{2}$, respectively, three and two orders of magnitude higher than the value obtained here for $(\mathrm{PEA})_{2} \mathrm{PbI}_{4}$. This and the linearity of the dephasing rates over a wide range of excitation densities [13] highlights the substantial screening of the exciton-exciton interactions in these 2D perovskites. This is especially surprising given the high biexciton binding energy [7], another characteristic that (PEA) ${ }_{2} \mathrm{PbI}_{4}$ shares with TMDC monolayers $[15,16]$. We will return to these differences in $\Delta$ below.

We now turn to the temperature dependence of $\gamma$ to investigate exciton-phonon interactions. The measured $\gamma$ for excitons $\mathrm{A}, \mathrm{A}^{\prime}$, and $\mathrm{B}$ as a function of temperature are presented in Figs. 3(d)-3(f), respectively. We fit this data with

$$
\gamma_{\mathrm{ph}}(T)=\gamma_{T=0}+\alpha_{\mathrm{LO}}\left[\frac{1}{\exp \left(E_{\mathrm{LO}} / k_{B} T\right)-1}\right],
$$


which assumes that line broadening arises from scattering of excitons with a single thermally populated phonon mode of effective energy $E_{\mathrm{LO}}$ with an effective interaction parameter $\alpha_{\mathrm{LO}}$. The parameters representing the best fit for each exciton are shown in Figs. 3(d) and 3(f). This model approximates well the behavior of excitons $\mathrm{A}$ and $\mathrm{B}$ but fails to reproduce that of exciton $\mathrm{A}^{\prime}$, an indication of the presence of complex lattice interactions as elucidated in our recent works [2,3,7]. To compare the strength of the obtained interaction parameters with those of other semiconductors, we obtain the linear interaction parameter $\Delta_{\mathrm{ph}}$ by taking the low-temperature limit of Eq. (5), yielding $\Delta_{\mathrm{ph}}=\alpha_{\mathrm{ph}} k_{B} / E_{\mathrm{LO}}$. This gives linear interaction parameters of $70 \pm 20$ and $130 \pm 10 \mu \mathrm{eV} / \mathrm{K}$ for excitons $\mathrm{A}$ and $\mathrm{B}$, respectively. These are almost an order of magnitude larger than those in two-dimensional quantum wells [17,18] and curiously comparable to monolayers of (covalent) TMDCs [4], given the ionic character of the lattice and the resulting strong electron-phonon coupling.

Despite the strong polaronic dressing of the excitons with distinct yet multiple phonon modes [3], here we find that only one phonon effective energy dominates the scattering process via weak interactions. Exciton A, dressed primarily by the in-plane lattice modes [3], interacts with optical phonons at $6.5 \mathrm{meV}$, which correspond to lattice modes within the inorganic lead-halide network. Exciton B, which is predominantly dressed by out-of-plane lattice modes [3] (see also Ref. [19]), interacts with higher energy phonons at $21 \mathrm{meV}$, which we have identified to have large contributions from the motion of the organic cation, particularly from the $\pi$ $\pi$ motion of the phenyl groups [20]. This behavior only highlights the complex phonon coupling scenario which may be beyond a conventional Fröhlich formalism and calls for further experimental and theoretical investigations. Two experimental observations presented in this work stand out-the remarkably low interexciton scattering rates and relatively weak exciton-phonon scattering. Coulomb interactions that are at the heart of both these scattering events, are, however, expected to be substantially enhanced in two-dimensional systems as evidenced in the case of TMDCs where excitons exhibit comparable Bohr radii [4]. We consider that polaronic effects [21] are responsible in the case of 2D Hybrid OrganicInorganic Perovskite (HOIPs) in screening the multiparticle Coulomb interactions. Such a mechanism has been invoked in the case of bulk 3D perovskites to explain slow cooling of hot carriers [22] and long carrier lifetimes [23,24], and analogous comparisons can be drawn in their 2D counterparts. Excitons reorganize the lattice along well-defined configurational coordinates [3], which dresses the exciton with a phonon cloud. Although we currently do not have a reliable estimate of the spatial extent of this deformation, i.e., the polaron radius, based on the polaron coupling constants in leadhalide perovskites [25], we may hypothesize that the radius is much larger than the exciton radius. The presence of such a lattice deformation surrounding the exciton can effectively shield it from collisional broadening due to interexcitonic interactions as evidenced by the relatively low $\gamma_{\text {EID }}$. At higher densities, we expect a high probability of multiple excitons within the polaron radius, in which case biexcitons may be populated given their high binding energy [7]. We have also noted recently [21] that strong Fröhlich-like exciton-phonon scattering is possible only in materials with exciton binding energy $\left(E_{B}\right)$ comparable to $E_{\mathrm{LO}}$ or with vast difference in the effective masses of the electrons and holes that make up the exciton [26]. With $E_{B}$ orders of magnitude higher than the soft lattice phonon energies, 2D HOIPs are thus expected to exhibit low elastic exciton-phonon scattering rates and in fact they are comparable to that of TMDCs [4] with similar $E_{B}$ as noted earlier. That being said, there is unambiguous presence of polaronic effects in 2D HOIPs [3] and a possible interplay between long-range (phonon) and short-range lattice coupling that may render Fröhlich mechanism insufficient to describe the exciton-lattice interactions.

Lastly, we note that the zero-density $\left(\gamma_{0}\right)$ and the zerotemperature $\left(\gamma_{T=0}\right)$ dephasing widths for all the excitonic transitions are between 2 and $3 \mathrm{meV}$, which corresponds to a pure dephasing time $T_{2}^{*}=\hbar / \gamma_{0, T=0} \approx 500 \mathrm{fs}$. Time-resolved photoluminescence measurements performed at $5 \mathrm{~K}$ have revealed a lower limit for the exciton lifetime to be around $100 \mathrm{ps}$ [27], suggesting a radiative width $\gamma_{\text {rad }} \sim 0.04 \mathrm{meV}$, which is much lower than the measured lower limit $\gamma_{0, T=0}$. This clearly demonstrates the presence of an additional dephasing mechanism in addition to interexciton and phonon scattering, possibly due to the defective nature of the polycrystalline film, the presence of other degenerate dark states which excitons scatter in and out of, or via low-energy acoustic phonons unresolved in the current experiment. In fact, the rephasing line shape with population waiting time evolves in a complex manner that suggests a role of dark states in both population and coherence dynamics; these dynamics will be discussed in a separate publication. We note that similar subpicosecond dephasing times as those reported in this work were measured at low temperature for photocarriers in $\mathrm{CH}_{3} \mathrm{NH}_{3} \mathrm{PbI}_{3}$, which were noticed to be a factor of $\approx 3$ times longer than in bulk GaAs [28]. This was ascribed to weaker Coulomb interactions in that perovskite, limiting the role of excitationinduced dephasing effects compared to III-V semiconductors. In Ref. [28], it was speculated that dynamic large polarons account for the relatively long photocarrier dephasing time. We point out that these relatively slow dephasing rates are still much too fast for quantum optoelectronics applications such as single-photon emitters [29], which require coherence times approaching the radiative lifetime.

In a general sense, multiexciton interactions are determined by an interplay of Coulomb forces, and in ionic crystalline systems, the role of the lattice in mediating them is of fundamental importance. We have focused on the comparison of elastic scattering processes of spectrally distinct excitons within the excitation fine structure, manifested via the dephasing rate. In a previous publication, we have reported high biexciton binding energies in this material [7] and importantly that biexcitons display distinct spectral structure. We highlight that in Ref. [7], the biexciton binding energies of excitons $\mathrm{A}$ and $\mathrm{B}$ appear to be different, and exciton $\mathrm{A}$ displays clear evidence of repulsive interactions in the twoquantum, two-dimensional spectral correlation map. Such interactions might give rise to inelastic scattering of exciton A, perhaps related to Auger recombination [5]. These overlapping dynamics would be deterministic in biexciton lasers [30], for example, if these devices were to be rigorously implemented. The extent to which the spectral scattering rates 
depend on spectral structure might also determine the dynamics of exciton polaritons in semiconductor microcavities, in which quantum fluids are formed by polariton-polariton inelastic scattering [31]. It has been demonstrated that polaritonpolariton interactions in 2D lead-halide perovskites are strong [32], such that the $\approx 0.5$-ps intrinsic dephasing times reported here are long compared to Rabi oscillation periods given $\gtrsim 150$-meV Rabi splittings [33]. In the search for roomtemperature polartion condensates, it is important to know how polaronic effects control exciton many-body dephasing dynamics and how hybrid perovskites differ in this respect from other two-dimensional candidate semiconductor systems such as monolayer TMDCs [4,14]. If polaronic effects mitigate excitation-induced dephasing in 2D hybrid perovskites, then the quantum dynamics in a vast range of structurally diverse derivatives merit profound experimental and theoretical investigation.

A.R.S.K. acknowledges funding from EU Horizon 2020 via a Marie Sklodowska Curie Fellowship (Global) (Project No. 705874). This work is partially supported by the National Science Foundation (Award No. 1838276). C.S. acknowledges support from the School of Chemistry and Biochemistry and the College of Science of Georgia Institute of Technology.
[1] K. Gauthron, J.-s. Lauret, L. Doyennette, G. Lanty, A. A. Choueiry, S. J. Zhang, L. Largeau, O. Mauguin, J. Bloch, and E. Deleporte, Opt. Express 18, 5912 (2010).

[2] S. Neutzner, F. Thouin, D. Cortecchia, A. Petrozza, C. Silva, and A. R. Srimath Kandada, Phys. Rev. Mat. 2, 064605 (2018).

[3] F. Thouin, D. A. Valverde-Chávez, C. Quarti, D. Cortecchia, I. Bargigia, D. Beljonne, A. Petrozza, C. Silva, and A. R. Srimath Kandada, Nat. Mater. 18, 349 (2019).

[4] G. Moody, C. Kavir Dass, K. Hao, C.-H. Chen, L.-J. Li, A. Singh, K. Tran, G. Clark, X. Xu, G. Berghäuser, E. Malic, A. Knorr, and X. Li, Nat. Commun. 6, 8315 (2015).

[5] G. Xing, B. Wu, X. Wu, M. Li, B. Du, Q. Wei, J. Guo, E. K. L. Yeow, T. C. Sum, and W. Huang, Nat. Commun. 8, 14558 (2017).

[6] D. B. Turner and K. A. Nelson, Nature (London) 466, 1089 (2010).

[7] F. Thouin, S. Neutzner, D. Cortecchia, V. A. Dragomir, C. Soci, T. Salim, Y. M. Lam, R. Leonelli, A. Petrozza, A. R. S. Kandada, and C. Silva, Phys. Rev. Mat. 2, 034001 (2018).

[8] K. Abdel-Baki, F. Boitier, H. Diab, G. Lanty, K. Jemli, F. Lédée, D. Garrot, E. Deleporte, and J. S. Lauret, J. Appl. Phys. 119, 064301 (2016).

[9] See Supplemental Material at http://link.aps.org/supplemental/ 10.1103/PhysRevResearch.1.032032 for a description of experimental methods, temperature-dependent absorption spectra, fluence-dependent nonlinear coherent spectra, and temperaturedependent nonlinear coherent spectra.

[10] M. E. Siemens, G. Moody, H. Li, A. D. Bristow, and S. T. Cundiff, Opt. Express 18, 17699 (2010).

[11] A. D. Bristow, T. Zhang, M. E. Siemens, S. T. Cundiff, and R. P. Mirin, J. Phys. Chem. B 115, 5365 (2011).

[12] The spectrum displayed in Fig. 1(c) was measured at a population waiting time of $20 \mathrm{fs}$, which is sufficiently short to be devoid of spectral dynamics but avoids coherent artifacts that result from ambiguous time ordering of the ultrashort pulses.

[13] C. Ciuti, V. Savona, C. Piermarocchi, A. Quattropani, and P. Schwendimann, Phys. Rev. B 58, 7926 (1998).

[14] E. W. Martin, J. Horng, H. G. Ruth, E. Paik, M.-H. Wentzel, H. Deng, and S. T. Cundiff, arXiv:1810.09834 [cond-mat.mtrlsci].
[15] Y. You, X.-X. Zhang, T. C. Berkelbach, M. S. Hybertsen, D. R. Reichman, and T. F. Heinz, Nat. Phys. 11, 477 (2015).

[16] I. Kylanpaa and H.-P. Komsa, Phys. Rev. B 92, 205418 (2015).

[17] A. Honold, L. Schultheis, J. Kuhl, and C. W. Tu, Phys. Rev. B 40, 6442 (1989).

[18] H. P. Wagner, A. Schätz, R. Maier, W. Langbein, and J. M. Hvam, Phys. Rev. B 56, 12581 (1997).

[19] A. Fieramosca, L. De Marco, M. Passoni, L. Polimeno, A. Rizzo, B. L. T. Rosa, G. Cruciani, L. Dominici, M. De Giorgi, G. Gigli, L. C. Andreani, D. Gerace, D. Ballarini, and D. Sanvitto, ACS Photon. 5, 4179 (2018).

[20] V. A. Dragomir, S. Neutzner, C. Quarti, D. Cortecchia, A. Petrozza, S. Roorda, D. Beljonne, R. Leonelli, A. R. S. Kandada, and C. Silva, arXiv:1812.05255 [cond-mat.mtrl-sci].

[21] See A. R. Srimath Kandada and C. Silva, arXiv:1908.03909 [cond-mat.mtrl-sci], for our perspective on exciton polarons as primary quasi-particles in these materials. for our perspective on exciton polarons as primary quasiparticles in these materials.

[22] P. P. Joshi, S. F. Maehrlein, and X. Zhu, Adv. Mater. 31, 1803054 (2019).

[23] C. Wehrenfennig, M. Liu, H. J. Snaith, M. B. Johnston, and L. M. Herz, Energy Environ. Sci. 7, 2269 (2014).

[24] T. M. Brenner, D. A. Egger, A. M. Rappe, L. Kronik, G. Hodes, and D. Cahen, J. Phys. Chem. Lett. 6, 4754 (2015).

[25] K. Miyata, D. Meggiolaro, M. T. Trinh, P. P. Joshi, E. Mosconi, S. C. Jones, F. De Angelis, and X.-Y. Zhu, Sci. Adv. 3, e1701217 (2017).

[26] M. Ueta, H. Kanzaki, K. Kobayashi, Y. Toyozawa, and E. Hanamura, Excitonic Processes in Solids, Springer Series in Solid-State Sciences, Vol. 60 (Springer Science \& Business Media, Berlin, 2012).

[27] D. B. Straus, S. Hurtado Parra, N. Iotov, J. Gebhardt, A. M. Rappe, J. E. Subotnik, J. M. Kikkawa, and C. R. Kagan, J. Am. Chem. Soc. 138, 13798 (2016)

[28] S. A. March, D. B. Riley, C. Clegg, D. Webber, X. Liu, M. Dobrowolska, J. K. Furdyna, I. G. Hill, and K. C. Hall, ACS Photon. 4, 1515 (2017).

[29] H. Utzat, W. Sun, A. E. Kaplan, F. Krieg, M. Ginterseder, B. Spokoyny, N. D. Klein, K. E. Shulenberger, C. F. Perkinson, M. V. Kovalenko et al., Science 363, 1068 (2019). 
[30] E. P. Booker, M. B. Price, P. J. Budden, H. Abolins, Y. del Valle-Inclan Redondo, L. Eyre, I. Nasrallah, R. T. Phillips, R. H. Friend, F. Deschler et al., Adv. Opt. Mater. 6, 1800616 (2018).

[31] I. Carusotto and C. Ciuti, Rev. Mod. Phys. 85, 299 (2013).
[32] A. Fieramosca, L. Polimeno, V. Ardizzone, L. De Marco, M. Pugliese, V. Maiorano, M. De Giorgi, L. Dominici, G. Gigli, D. Gerace et al., arXiv:1811.04041 [physics.optics].

[33] A. Brehier, R. Parashkov, J. S. Lauret, and E. Deleporte, Appl. Phys. Lett. 89, 171110 (2006). 